\title{
APPLICATION OF UNIFORM ASYMPTOTICS TO THE FIFTH PAINLEVÉ TRANSCENDANT
}

\author{
YOUMIN LU and ZHOUDE SHAO
}

Received 30 August 2001

We apply the uniform asymptotics method to the fifth Painleve transcendants, find its asymptotics of the form $y=-1+t^{-1 / 2} A(t)$ as $t \rightarrow \infty$ along the positive $t$-axis, and obtain the corresponding monodromy data.

2000 Mathematics Subject Classification: 34E05.

1. Introduction. We study the general fifth Painlevé equation

$$
\begin{aligned}
\frac{d^{2} y}{d t^{2}}= & \left(\frac{1}{2 y}+\frac{1}{y-1}\right)\left(\frac{d y}{d t}\right)^{2}-\frac{1}{t} \frac{d y}{d t} \\
& +\frac{(y-1)^{2}}{t^{2}}\left(\alpha y+\frac{\beta}{y}\right)+\frac{\gamma y}{t}+\frac{\delta y(y+1)}{y-1},
\end{aligned}
$$

where $\alpha, \beta, \gamma$, and $\delta$ are parameters, and its solution of the form

$$
\begin{aligned}
y(t) & =-1+4 t^{-1 / 2} A(t), \\
y^{\prime}(t) & =-2 t^{-3 / 2} A(t)+4 t^{-1 / 2} A^{\prime}(t)=4 t^{-1 / 2} A^{\prime}(t)+O\left(t^{-3 / 2}\right),
\end{aligned}
$$

with $A(t)=O(1)$ as $t \rightarrow \infty$.

The fifth Painlevé equation (1.1) can be obtained as the compatibility condition of the following linear systems of equations (see [2, 3]):

$$
\begin{aligned}
& Y_{z}^{\prime}(z)=\left(\begin{array}{cc}
\frac{t}{2}+\frac{2 v+\theta_{0}}{2 z}-\frac{w}{z-1} & -\frac{u\left(v+\theta_{0}\right)}{z}+\frac{u y\left(2 w-\theta_{1}\right)}{2(z-1)} \\
\frac{v}{u z}-\frac{2 w+\theta_{1}}{2 u y(z-1)} & -\frac{t}{2}-\frac{2 v+\theta_{0}}{2 z}+\frac{w}{z-1}
\end{array}\right) Y(z), \\
& Y_{t}^{\prime}(z)=\left(\begin{array}{cc}
\frac{1}{2} & \frac{u}{z}\left[v+\theta_{0}-y\left(w-\frac{\theta_{1}}{2}\right)\right] \\
\frac{1}{u z}\left[v-\frac{1}{y}\left(w+\frac{\theta_{1}}{2}\right)\right] & -\frac{1}{2}
\end{array}\right) Y(z),
\end{aligned}
$$

where

$$
\begin{aligned}
w & =v+\frac{1}{2}\left(\theta_{0}+\theta_{\infty}\right), \\
t \frac{d y}{d t} & =t y-2 v(y-1)^{2}-\frac{1}{2}(y-1)\left[\left(\theta_{0}-\theta_{1}+\theta_{\infty}\right) y-\left(3 \theta_{0}+\theta_{1}+\theta_{\infty}\right)\right], \\
t \frac{d u}{d t} & =u\left\{-2 v-\theta_{0}+y\left[v+\frac{1}{2}\left(\theta_{0}-\theta_{1}+\theta_{\infty}\right)\right]+\frac{1}{y}\left[v+\frac{1}{2}\left(\theta_{0}+\theta_{1}+\theta_{\infty}\right)\right]\right\},
\end{aligned}
$$


with

$$
\alpha=\frac{1}{2}\left(\frac{\theta_{0}-\theta_{1}+\theta_{\infty}}{2}\right)^{2}, \quad \beta=-\frac{1}{2}\left(\frac{\theta_{0}-\theta_{1}-\theta_{\infty}}{2}\right)^{2}, \quad \gamma=1-\theta_{0}-\theta_{1}, \quad \delta=-\frac{1}{2} .
$$

The canonical solutions of system (1.3) are defined in [2] by

$$
\begin{gathered}
-\frac{3 \pi}{2}+k \pi \leq \arg \lambda<-\frac{\pi}{2}+k \pi, \\
Y_{k}(\lambda) \sim \hat{Y}_{\infty}(\lambda) e^{(t \lambda / 2-\log \lambda) \sigma_{3}},
\end{gathered}
$$

where $\sigma_{3}=\left(\begin{array}{cc}1 & 0 \\ 0 & -1\end{array}\right)$, and the Stokes multiplier $G_{1}$ is defined in [2] by

$$
Y_{2}(\lambda)=Y_{1}(\lambda) G_{1}
$$

where $G_{1}=\left(\begin{array}{ll}1 & 0 \\ s & 1\end{array}\right)$ and its entry $s$ is independent of $t$ and $y$.

2. Reduction of the problem. Generally, if $(d / d z)\left(\begin{array}{l}Y_{1} \\ Y_{2}\end{array}\right)=\left(\begin{array}{cc}A & B \\ C & -A\end{array}\right)\left(\begin{array}{l}Y_{1} \\ Y_{2}\end{array}\right), \phi=B^{-1 / 2} Y_{1}$, and $\psi=C^{-1 / 2} Y_{2}$, then

$$
\begin{aligned}
& \frac{d^{2} \phi}{d z^{2}}=\left(A^{2}+B C+A^{\prime}-B^{\prime} B^{-1} A+\frac{3}{4} B^{-2} B^{\prime 2}-\frac{1}{2} B^{-1} B^{\prime \prime}\right) \phi \\
& \frac{d^{2} \psi}{d z^{2}}=\left(A^{2}+B C-A^{\prime}+C^{\prime} C^{-1} A+\frac{3}{4} C^{-2} C^{\prime 2}-\frac{1}{2} C^{-1} C^{\prime \prime}\right) \psi
\end{aligned}
$$

We first apply the transformation

$$
\hat{Y}=\left(\begin{array}{ll}
1 & i \\
i & 1
\end{array}\right) u^{-(1 / 2) \sigma_{3}} Y
$$

to system (1.3) to get

$$
\frac{d \hat{Y}}{d z}=\frac{1}{2}\left(\begin{array}{cc}
L & N \\
M & -L
\end{array}\right) \hat{Y}
$$

where

$$
\begin{aligned}
& L=\frac{i\left(2 v+\theta_{0}\right)}{z}-\frac{i(1 / y+y) w+i(1 / y-y)\left(\theta_{1} / 2\right)}{z-1}, \\
& M=\frac{i\left(2 v+\theta_{0}\right)-\theta_{0}}{z}+\frac{(y-1 / y-2 i) w-(y+1 / y)\left(\theta_{1} / 2\right)}{z-1}+i t, \\
& N=-\frac{i\left(2 v+\theta_{0}\right)+\theta_{0}}{z}+\frac{(y-1 / y+2 i) w-(y+1 / y)\left(\theta_{1} / 2\right)}{z-1}-i t .
\end{aligned}
$$


Applying (2.1) to (2.3), we get

$$
\begin{aligned}
& \frac{d^{2} \phi}{d z^{2}}=\left\{\left(\frac{t}{2}+\frac{2 v+\theta_{0}}{2 z}-\frac{w}{z-1}\right)^{2}+\left[-\frac{u\left(v+\theta_{0}\right)}{z}+\frac{u y\left(2 w-\theta_{1}\right)}{2(z-1)}\right]\left[\frac{v}{u z}-\frac{2 w+\theta_{1}}{2 u y(z-1)}\right]\right. \\
& -\frac{i\left(2 v+\theta_{0}\right)}{2 z^{2}}+\frac{i\left(\frac{1}{y}+y\right) w+i\left(\frac{1}{y}-y\right) \frac{\theta_{1}}{2}}{2(z-1)^{2}} \\
& -\frac{\left[\frac{2 i v+(i+1) \theta_{0}}{z^{2}}-\frac{\left(y-\frac{1}{y}+2 i\right) w-\left(y+\frac{1}{y}\right) \frac{\theta_{1}}{2}}{(z-1)^{2}}\right] \frac{L}{2}}{N} \\
& +\frac{3}{4} \frac{\left[\frac{2 i v+(i+1) \theta_{0}}{z^{2}}-\frac{\left(y-\frac{1}{y}+2 i\right) w-\left(y+\frac{1}{y}\right) \frac{\theta_{1}}{2}}{(z-1)^{2}}\right]^{2}}{N^{2}} \\
& \left.-\frac{-\frac{2 i v+(i+1) \theta_{0}}{z^{3}}+\left(y-\frac{1}{y}+2 i\right) w-\left(y+\frac{1}{y}\right) \frac{\theta_{1}}{2}}{N}\right\} \phi .
\end{aligned}
$$

Now, using (1.6), the following asymptotics can be obtained:

$$
\begin{aligned}
& v(t)=-\frac{1}{8} t-\frac{1}{2} t^{1 / 2} A^{\prime}(t)+\frac{1}{2} A^{2}(t)-2 A(t) A^{\prime}(t)-\frac{1}{2} \theta_{0}-\frac{1}{4} \theta_{\infty}+O\left(t^{-1 / 2}\right), \\
& u(t)=C e^{t / 2}\left(1+O\left(t^{-1 / 2}\right)\right) .
\end{aligned}
$$

Substituting (2.6) into (2.5), we get the following second-order equation:

$$
\begin{aligned}
\frac{d^{2} \phi}{d z^{2}}=-t^{2}\{ & -\frac{(2 z-1)^{2}}{16 z(z-1)}-t^{-1}\left[-\frac{(2 z-1) \theta_{\infty}}{4 z(z-1)}-\frac{A^{2}+4 A^{\prime 2}}{4 z(z-1)}+\frac{i\left(2 z^{2}-2 z+1\right)}{8 z^{2}(z-1)^{2}}\right. \\
& \left.\left.+\frac{i(2 z-1)\left[(i t / 4)(2 z-1)+t^{1 / 2} A z^{2}\right]}{8 z^{2}(z-1)^{2}\left[-i t^{1 / 2} A^{\prime}-t^{1 / 2} A z-i t(z-1 / 2)^{2}\right]}\right]+O\left(t^{-3 / 2}\right)\right\} \phi \\
= & -t^{2} F(z, t) \phi .
\end{aligned}
$$

Equation (2.7) has two turning points

$$
z_{j}=\frac{1}{2} \pm t^{-1 / 2} \sqrt{A^{2}+4 A^{\prime 2}+i}(1+o(1)), \quad j=1,2
$$


which merge to $1 / 2$ as $t \rightarrow \infty$, and Stokes directions

$$
\operatorname{Re}(\sqrt{z(z-1)})=0
$$

Now, we define a constant $\alpha$ by

$$
\frac{1}{2} \pi i \alpha^{2}=\int_{-\alpha}^{\alpha}\left(\tau^{2}-\alpha^{2}\right)^{1 / 2} d \tau=\int_{z_{1}}^{z_{2}} F^{1 / 2}(z, t) d z
$$

and a new variable $\zeta$ by

$$
\int_{\alpha}^{\zeta}\left(\tau^{2}-\alpha^{2}\right)^{1 / 2} d \tau=\int_{z_{1}}^{z} F^{1 / 2}(s, t) d s
$$

Using [1, Theorem 1], we have the following theorem.

THEOREM 2.1. Given any solution $\phi$ of (2.7), there exist constants $c_{1}$ and $c_{2}$ such that, uniformly for $z$ on the Stokes curve, as $t \rightarrow \infty$,

$$
\left(\frac{\zeta^{2}-\alpha^{2}}{F(z, t)}\right)^{-1 / 4} \phi(z, t)=\left\{\left(c_{1}+o(1)\right) D_{v}\left(e^{\pi i / 4} \sqrt{2 t} \zeta\right)+\left(c_{2}+o(1)\right) D_{-v-1}\left(e^{-\pi i / 4} \sqrt{2 t} \zeta\right)\right\},
$$

where $v=-1 / 2+(1 / 2)$ it $\alpha^{2}$ and $D_{v}(z), D_{-v-1}(z)$ are solutions of the parabolic cylinder equation.

\section{Monodromy data and asymptotics}

THEOREM 3.1. For large $t$ and $z$,

$$
\begin{aligned}
\frac{1}{2} \zeta^{2}- & \frac{A^{2}+4 A^{\prime 2}+i}{2 t} \log \zeta+o\left(t^{-1}\right) \\
= & \frac{i z}{2}-\frac{i \theta_{\infty}}{2 t} \log (4 z)+\frac{i}{2 t} \log \frac{2 i t^{1 / 2}}{2 i A^{\prime}+A}+\frac{1-i}{4}-\frac{\pi \theta_{\infty}}{4 t} \\
& -\frac{\pi i \alpha^{2}}{4}+o\left(t^{-1}\right)+O\left(z^{-1}\right) .
\end{aligned}
$$

PRoof. Carrying out the integration on the left-hand side of (2.11), we have

$$
\frac{1}{2} \zeta^{2}-\frac{\alpha^{2}}{2} \log (2 \zeta)-\frac{\alpha^{2}}{4}+\frac{\alpha^{2}}{2} \log \alpha+O\left(\alpha^{4} \zeta^{-2}\right)=\int_{z_{1}}^{z} F^{1 / 2}(s, t) d s
$$

Because we are going to calculate the higher-order part of the right-hand side, we will simply ignore the lower-order part in $F(z, t)$, and split the right-hand side into two integrals

$$
\int_{z_{1}}^{z} F^{1 / 2}(s, t) d s=\left(\int_{z_{1}}^{z^{*}}+\int_{z^{*}}^{z}\right) F^{1 / 2}(x, t) d x=I_{1}+I_{2},
$$


where $z^{*}=1 / 2+T t^{-1 / 2}$ and $T$ is a large parameter to be specified later. Using the substitution

$$
x-\frac{1}{2}=s t^{-1 / 2}
$$

$I_{1}$ can be evaluated as follows:

$$
\begin{aligned}
I_{1}= & \frac{1}{t} \int_{\sqrt{A^{2}+4 A^{\prime 2}}}^{T}\left(\sqrt{s^{2}-\left(A^{2}+4 A^{\prime 2}+i\right)}+o(1)\right) d s \\
= & \frac{T^{2}}{2 t}-\frac{A^{2}+4 A^{\prime 2}+i}{4 t}-\frac{A^{2}+4 A^{\prime 2}+i}{2 t} \log (2 T) \\
& +\frac{A^{2}+4 A^{\prime 2}+i}{4 t} \log \left(A^{2}+4 A^{\prime 2}+i\right)+o\left(t^{-1}\right) .
\end{aligned}
$$

Using the formula

$$
\begin{aligned}
& \int \frac{2 a x+b}{\left(a x^{2}+b x+c\right) \sqrt{x^{2}-\frac{1}{4}}} d x \\
& =2 a \frac{\left(\operatorname{arctanh} \frac{a+2 b x-2 \sqrt{\left(b^{2}-4 a c\right)} x}{\sqrt{\left(2 b^{2}-2 b \sqrt{\left(b^{2}-4 a c\right)}-4 a c-a^{2}\right)} \sqrt{\left(4 x^{2}-1\right)}}\right)}{\sqrt{\sqrt{\left(2 b^{2}-2 b \sqrt{\left(b^{2}-4 a c\right)}-4 a c-a^{2}\right)}}} \\
& +2 a \frac{a+2 b x+2 \sqrt{\left(b^{2}-4 a c\right)} x}{\sqrt{\left(2 b^{2}+2 b \sqrt{\left(b^{2}-4 a c\right)}-4 a c-a^{2}\right)} \sqrt{\left(4 x^{2}-1\right)}}
\end{aligned}
$$

we find the asymptotic expression of $I_{2}$

$$
\begin{aligned}
& I_{2}=\int_{z^{*}}^{z}\left\{-\frac{(2 s-1)^{2}}{16 s(s-1)}-t^{-1}[\right.-\frac{(2 s-1) \theta_{\infty}}{4 s(s-1)}-\frac{A^{2}+4 A^{\prime 2}}{4 s(s-1)}+\frac{i\left(2 s^{2}-2 s+1\right)}{8 s^{2}(s-1)^{2}} \\
&\left.\left.-\frac{i(2 s-1)\left[(1 / 2) i t^{1 / 2}(s-1 / 2)+A s^{2}\right]}{8 z^{2}(s-1)^{2}\left[i A^{\prime}+A s+i t^{1 / 2}(s-1 / 2)^{2}\right]}\right]\right\}^{1 / 2} d s \\
&=\int_{z^{*}}^{z} \frac{i(2 s-1)}{4 \sqrt{s(s-1)}\left\{1+\frac{16 s(s-1)}{t(2 s-1)^{2}}[\right.}\left[-\frac{(2 s-1) \theta_{\infty}}{4 s(s-1)}-\frac{A^{2}+4 A^{\prime 2}}{4 s(s-1)}+\frac{i\left(2 s^{2}-2 s+1\right)}{8 s^{2}(s-1)^{2}}\right. \\
&\left.\left.+\frac{i(2 s-1)\left[(i t / 4)(2 s-1)+t^{1 / 2} A s^{2}\right]}{8 s^{2}(s-1)^{2}\left[-i t / 4-t^{1 / 2} A^{\prime}-t^{1 / 2} A s-i t s(s-1)\right]}\right]\right\}^{1 / 2} d s
\end{aligned}
$$




$$
\begin{aligned}
=\int_{z^{*}}^{z}\left[\frac{i(2 s-1)}{4 \sqrt{s(s-1)}}-\frac{i \theta_{\infty}}{2 t \sqrt{s(s-1)}}-\frac{i\left(A^{2}+4 A^{\prime 2}+i\right)}{2 t(2 s-1) \sqrt{s(s-1)}}\right. \\
\left.\quad-\frac{2 i t^{1 / 2}(s-1 / 2)+A}{4 t \sqrt{(s-1 / 2)^{2}-1 / 4}\left[i A^{\prime}+(1 / 2) A+A(s-1 / 2)+i t^{1 / 2}(s-1 / 2)^{2}\right]}\right] d z \\
+O\left(t^{-2} \int_{z^{*}}^{z} \frac{|d s|}{|2 s-1|^{3}}\right)+O\left(\frac{1}{z}\right) \\
=\frac{i z}{2}-\frac{i}{4}-\frac{i \theta_{\infty}}{2 t} \log (4 z)-\frac{i \pi\left(A^{2}+4 A^{\prime 2}+i\right)}{4 t}+\frac{1}{4}-\frac{T^{2}}{2 t}-\frac{\pi \theta_{\infty}}{4 t} \\
-\frac{A^{2}+4 A^{\prime 2}+i}{4 t} \log \frac{t}{T^{2}}+\frac{i}{2 t} \log \frac{2 i t^{1 / 2}}{2 i A^{\prime}+A+O\left(t^{-1 / 4}\right)}+O\left(z^{-1}\right) \\
+O\left(T^{-2} t^{-1}\right)+O\left(T^{4} t^{-2}\right) .
\end{aligned}
$$

Using definition (2.10) and setting $T=-\sqrt{A^{2}+4 A^{\prime 2}}$ in $I_{1}$, we have the following expression for $\alpha$ :

$$
\alpha^{2}=-\frac{A^{2}+4 A^{\prime 2}+i}{t}+o\left(t^{-1}\right) .
$$

Substituting (3.8) into (3.2), setting $T<t^{1 / 4}$, and combining it with (3.5) and (3.7), the theorem is proved.

Knowing [4] that

$$
D_{v}(z) \sim \begin{cases}z^{v} e^{-(1 / 4) z^{2}}, & \text { if }|\arg z|<\frac{3}{4} \pi, \\ z^{v} e^{-(1 / 4) z^{2}}-\frac{\sqrt{2 \pi}}{\Gamma(-v)} e^{i \pi v} z^{-v-1} e^{(1 / 4) z^{2}}, & \text { if } \arg z=\frac{3}{4} \pi,\end{cases}
$$

and $\arg \left(e^{\pi i / 4} \sqrt{2 t} \zeta\right) \sim \pi / 4$ when $z \rightarrow-\infty$, we can choose, as $z \rightarrow-\infty$,

$$
\begin{aligned}
\hat{Y}_{2}^{(11)}(z) \sim & z^{-\theta_{\infty} / 2} e^{t z / 2} \\
\sim & 2^{\theta_{\infty}} t^{1 / 4} \sqrt{2 i A^{\prime}+A} e^{-(\pi i / 4)\left(1 / 2+(3 i t / 2) \alpha^{2}\right)} e^{(1 / 4) t+i\left[t / 4-(1 / 4)\left(A^{2}+4 A^{\prime 2}\right) \log (2 t)+\theta_{\infty} / 4\right]} \\
& \times \zeta^{1 / 2} D_{v}\left(e^{\pi i / 4} \sqrt{2 t} \zeta\right) .
\end{aligned}
$$

Because $\arg \left(e^{\pi i / 4} \sqrt{2 t} \zeta\right) \sim 3 \pi / 4$ when $z \rightarrow \infty$, we have the following asymptotics for $\hat{Y}_{2}^{(11)}(z)$ as $z \rightarrow \infty$ :

$$
\begin{aligned}
\hat{Y}_{2}^{(11)}(z) \sim & z^{-\theta_{\infty} / 2} e^{t z / 2} \\
& -z^{\theta_{\infty} / 2} e^{-t z / 2} \frac{4^{\theta_{\infty}} \sqrt{\pi}\left(A^{2}+2 i A^{\prime 2}\right)}{\Gamma\left(1 / 2-(t i / 2) \alpha^{2}\right)} e^{(\pi i / 4)\left(2 t i \alpha^{2}-3\right)+(1 / 2) t+i\left[t / 2-(1 / 2)\left(A+4 A^{\prime 2}\right) \log (2 t)\right]} .
\end{aligned}
$$


By (1.9) and (2.2), the Stokes multiplier can be defined by $\hat{Y}_{2}=\hat{Y}_{1} G_{1}$. Therefore, the monodromy data is

$$
S=\frac{4^{\theta_{\infty}} \sqrt{\pi}\left(A+2 i A^{\prime}\right)}{\Gamma\left(1 / 2-(t i / 2) \alpha^{2}\right)} e^{(\pi i / 4)\left(2 t i \alpha^{2}-5\right)+i\left[t / 2-(1 / 2)\left(A+4 A^{\prime 2}\right) \log (2 t)\right]} .
$$

Taking the square of the absolute value of both sides of this equation, we find that $A^{2}+4 A^{\prime 2} \sim d^{2}$ where $d$ is a constant. Solving (3.12) for $A+2 i A^{\prime}$, we have

$$
A+2 i A^{\prime}=\left( \pm \sqrt{\left(A^{2}+4 A^{\prime 2}\right)}+O\left(t^{-1 / 2}\right)\right) e^{i\left[t / 2-(1 / 2)\left(A+4 A^{\prime 2}\right) \log (2 t)+\theta\right]} .
$$

Taking the real part and the imaginary part of (3.13), we obtain the following theorem.

THEOREM 3.2. Equation (1.1) has a solution with the following asymptotics:

$$
\begin{aligned}
& y(t) \sim-1+4 t^{-1 / 2}\left(d+O\left(t^{-1 / 2}\right)\right) \cos \left(\frac{t}{2}-\frac{1}{2} d^{2} \log (2 t)+\theta\right), \\
& y^{\prime}(t) \sim 2 t^{-1 / 2}\left(d+O\left(t^{-1 / 2}\right)\right) \sin \left(\frac{t}{2}-\frac{1}{2} d^{2} \log (2 t)+\theta\right), \quad \text { as } t \rightarrow \infty .
\end{aligned}
$$

\section{REFERENCES}

[1] A. P. Bassom, P. A. Clarkson, C. K. Law, and J. B. McLeod, Application of uniform asymptotics to the second Painlevé transcendent, Arch. Rational Mech. Anal. 143 (1998), no. 3, 241-271.

[2] A. S. Fokas, U. Muğan, and M. J. Ablowitz, A method of linearization for Painlevé equations: Painlevé IV, V, Phys. D 30 (1988), no. 3, 247-283.

[3] A. R. Its and V. Y. Novokshenov, The Isomonodromic Deformation Method in the Theory of Painlevé Equations, Lect. Notes in Math., vol. 1191, Springer-Verlag, Berlin, 1986.

[4] E. T. Whittaker and G. M. Watson, Modern Analysis, 4th ed., Cambridge University Press, Cambridge, 1927.

YOUMin LU: DePARTMENT OF MATHEMATICS AND COMPUTER SCIENCE, BLOOMSBURG UNIVERSITY, BLOOMSBURG, PA 17815, USA

Zhoude ShaO: Department of MAthematics, Millersville University, Millersville, PA 17551-0302, USA 


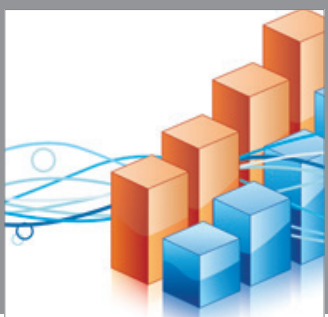

Advances in

Operations Research

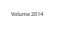

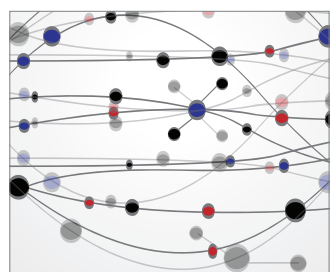

\section{The Scientific} World Journal
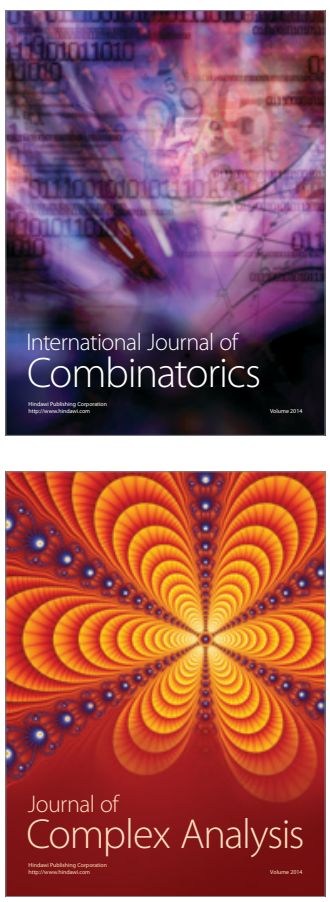

International Journal of

Mathematics and

Mathematical

Sciences
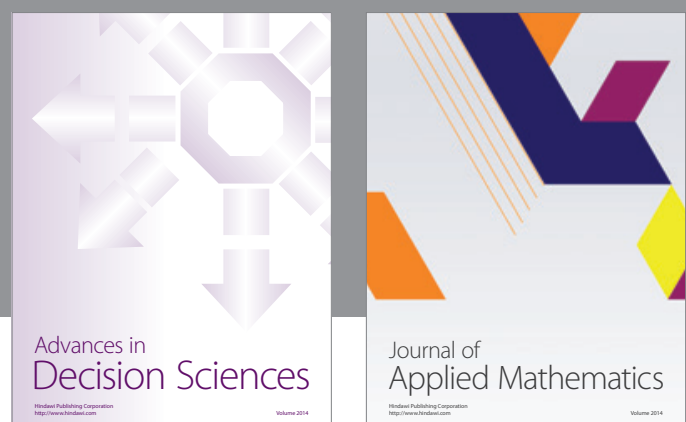

Journal of

Applied Mathematics
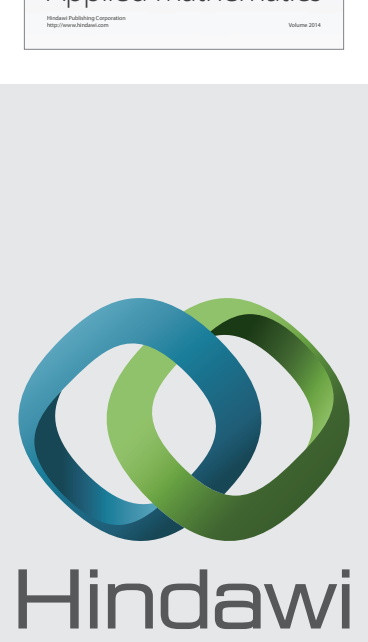

Submit your manuscripts at http://www.hindawi.com
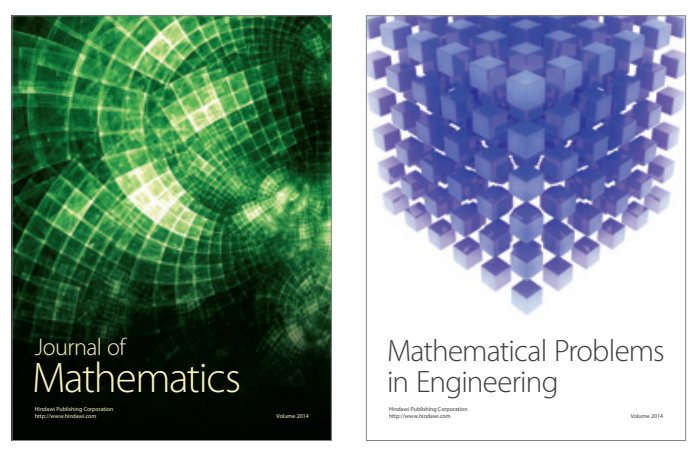

Mathematical Problems in Engineering
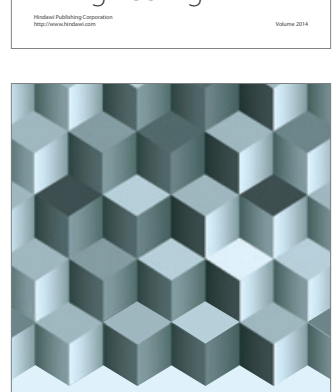

Journal of

Function Spaces
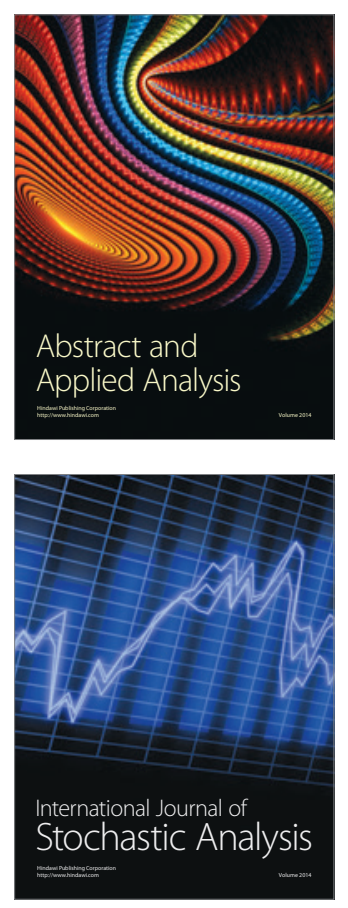

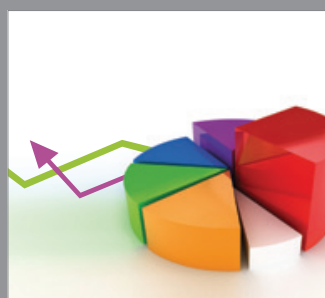

ournal of

Probability and Statistics

Promensencen
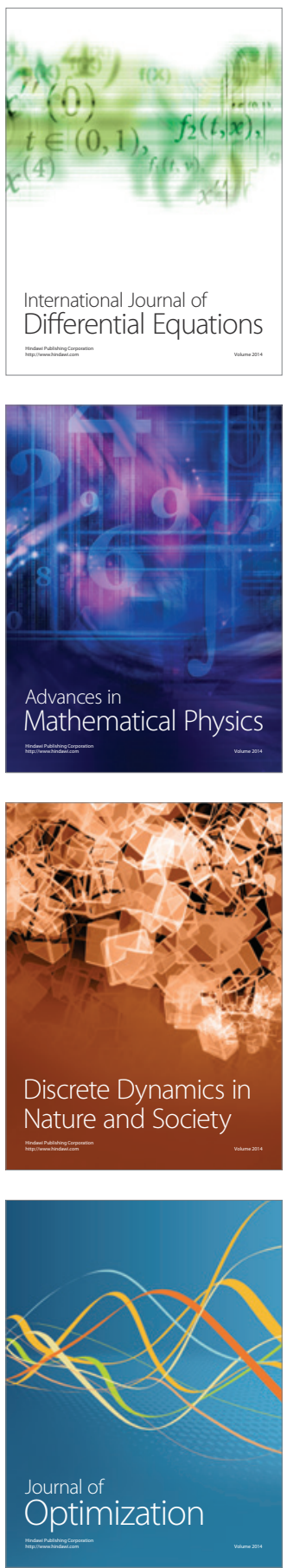